INNOVATIONS IN PRIMARY CARE

\title{
Using Patient-Entered Data to Supercharge Self-Management
}

Lynn Ho, MD, Jean Antonucci, MD

Ann Fam Med 2017;15:382. https://doi.org/10.1370/afm.2068.

\section{THE INNOVATION}

Patients' confidence in their ability to manage their own health conditions determines outcomes. We describe a low-burden method by which a practice of any size can measure and improve the health confidence of its population.

\section{WHO \& WHERE}

We are solo family doctors who have been developing our own practice quality paradigms in low-overhead, technologyenabled, insurance-based outpatient practices in North Kingstown, Rhode Island, and Farmington, Maine, since 2004-2005.

\section{HOW}

Since 2006 our practices have been gathering patient-entered data using a free internet tool, http://www.howsyourhealth.org (Supplemental Appendix 1), to monitor and improve individual and practice-wide patient health confidence levels among other metrics. High levels of patient confidence are clearly shown to correlate with decreased harms and cost savings., ${ }^{1,2}$ Additionally, high levels of patient confidence are directly correlated with improved population health metrics. ${ }^{3}$

The online tool includes a single question about health confidence: "How confident are you that you can control and manage most of your health problems?" Patients can respond very, somewhat, or not confident. We ask patients to complete the online survey in preparation for or during their preventive care visits and receive each patient's response via secure email before or during the visit, so we can address root causes for low confidence levels. For example, a common reason for low health confidence might be "I've tried, but haven't been able to lose weight." For such a reason, the response might involve running through a simple problem-solving algorithm (https:// howsyourhealth.com/pblmslv/), which could leave the patient with a plan to change a component of eating behavior. Small, sequential successes in behavior change improve self-management ability and confidence.

Conflicts of interest: authors report none.

\section{CORRESPONDING AUTHOR}

Lynn Ho, MD

North Kingstown Family Practice

320 Phillips St, Suite 102

North Kingstown, Rhode Island 02852

nkfpdoc@gmail.com
When we ask patients to take the online survey, we give them a practice code to enter, and results of surveys that include our practice codes are continuously aggregated for the practices on a secure server at no cost to the practices. We can monitor the practice-wide level of confidence with self-management at baseline, implement a plan to improve confidence practice wide, and then check to see the effect of the intervention on patient confidence levels. Using repeated iterations of the above technique, one of us (L.H.) succeeded in increasing practice-wide very confident ratings from the national norm of $50 \%$ in 2006 to $69 \%$ in 2017 . We have also used this continuously-aggregated, patient-entered health data to measure and then improve key primary care metrics such as continuity, efficiency, access, coordination and patient information (Supplemental Appendix 2).

\section{LEARNING}

Using patient-entered data to improve confidence in our practices has had 2 major effects. First, it has taught us that simple, low-cost, patient-centered methods exist for us and similar small practices to monitor and improve patient health confidence and other fundamental practice characteristics. Second, improvement of practice-wide health confidence has helped our practices better achieve health care's triple aim: better population health, better patient experience, and lower monthly expenditures per patient. Two key differences distinguish this quality improvement innovation from de facto quality rating schemata based on EHR documentation: (1) metrics are $100 \%$ based on patient input, not derived from practice attestation; (2) this process is remarkably low in both administrative burden and cost for practices, since patients enter the data into an automatically aggregating free online tool (Supplemental Appendix 3). Unfortunately, despite the advantages and ease of use of this combined approach to measurement and improvement, we have encountered resistance from regional payers and quality organizations when suggesting the use of patient entered data for quality measurement. Primary care physicians are forced to spend too much time gathering and reporting quality data when tools like How's Your Health? are available. We hope this article can spur uptake of this simple yet powerful method to measure quality and improve practice.

Key words: patient self-efficacy; surveys and questionnaires; practice quality improvement

Author affiliations, references, and 3 supplemental appendixes are available at http://www.annfammed.org/content/15/4/382I suppI/DC1. 